\title{
ВОЗМОЖНОСТЬ И ЭФФЕКТ ОТ ВНЕДРЕНИЯ АГЕНТНО-ОРИЕНТИРОВАННОЙ МОДЕЛИ В СИСТЕМУ УПРАВЛЕНИЯ ОРГАНИЗАЦИЕЙ
}

\author{
(C) 2020 Багрин Павел Петрович \\ Генеральный директор ООО «ТД «СмартВес», Россия, Мытищи \\ Государственный университет управления, Россия, Москва
}

В статье рассматривается вопрос применения агентно-ориентированных моделей в системе управления организацией. Рассмотрен алгоритм управления по отклонениям и выявлены возможности использования агентно-ориентированной модели на каждом из этапов. При выявлении места модели также выявлялся эффект от применения данного инструмента. Результаты исследования могут быть использованы при внедрении имитационных и агентно-ориентированных моделей в организациях, а также при разработке концепции модели.

Ключевые слова: Агентно-ориентированная модель, система управления, имитационная модель, управленческое решение, риски, обоснованность.

В настоящее время наблюдается сильный тренд в применении цифровых инструментов в управлении организациями. Ее необходимость обусловлена рядом факторов, среди которых наиболее выделяется значительный объем информации, который необходимо оценить управленцу для принятия обоснованных решений. Если необходимые данные не зафиксированы и не оцифрованы, решения приходится принимать на основе опыта, наблюдений, предположений, что неизбежно ведет к возникновению различных рисков, возникающих по причине не адекватной оценки как состояния объекта управления, так и условий, в которых он функционирует. Агентно-ориентированное моделирование (AОМ) является одним из инструментов, позволяющих повысить качество управленческих решений. Если в управлении техническими [2], [9], логистическими [4], [8] системами, анализе макросистем [1], [5], [6] этот инструмент широко применяется, то в управлении отдельными организациями этот инструмент еще не распространен. Применимость АОМ для корпоративных систем обоснована в статье [3], рассмотрим место такой модели в системе управления организации.

Для решения этой задачи рассмотрим целевую функцию системы управления - генерацию обоснованной и управляющей информации. Для определения места АОМ в системе управления рассмотрим алгоритм управления «по отклонениям» на рисунке 1 , сформированный Самосудовым М.В. в [7], и применение АОМ на каждом из его этапов. Можно выделить следующие эта- пы алгоритма управления:

- Сбор информации о состоянии системы,

- Есть ли отклонения требующие корректировки?

- Есть ли другие причины для корректировки?

- Определения направлений принятия решений,

- Формирование корректирующего воздействия,

- Проверка решения на обоснованность и влияние на устойчивость,

- Преобразование решения в действия.

Помимо указанных в схеме этапов можно выделить и предшествующий - формирование эталонного состояния системы. Рассмотрим последовательно каждый этап и выявим возможность применения АОМ на них.

Формирование эталонного состояния необходимо для определения того состояния системы, которое приведет к заданному состоянию - реализации целевой функции системы. Использование модели на этом этапе позволяет структурировать и формализовать информацию. Так, деятельность будет описываться перечнем действий, реализуемых разными агентами, формируется и формализуется эталонная ресурсная база, институциональная среда и т.д. Применение АOM на этом этапе определяет ту форму, в которой задается состояние - формы фиксации информации о состоянии. Использование компьютерной модели определяет требования к фиксации информации о состоянии, это должны быть оцифрованные параметры. Другими сло- 


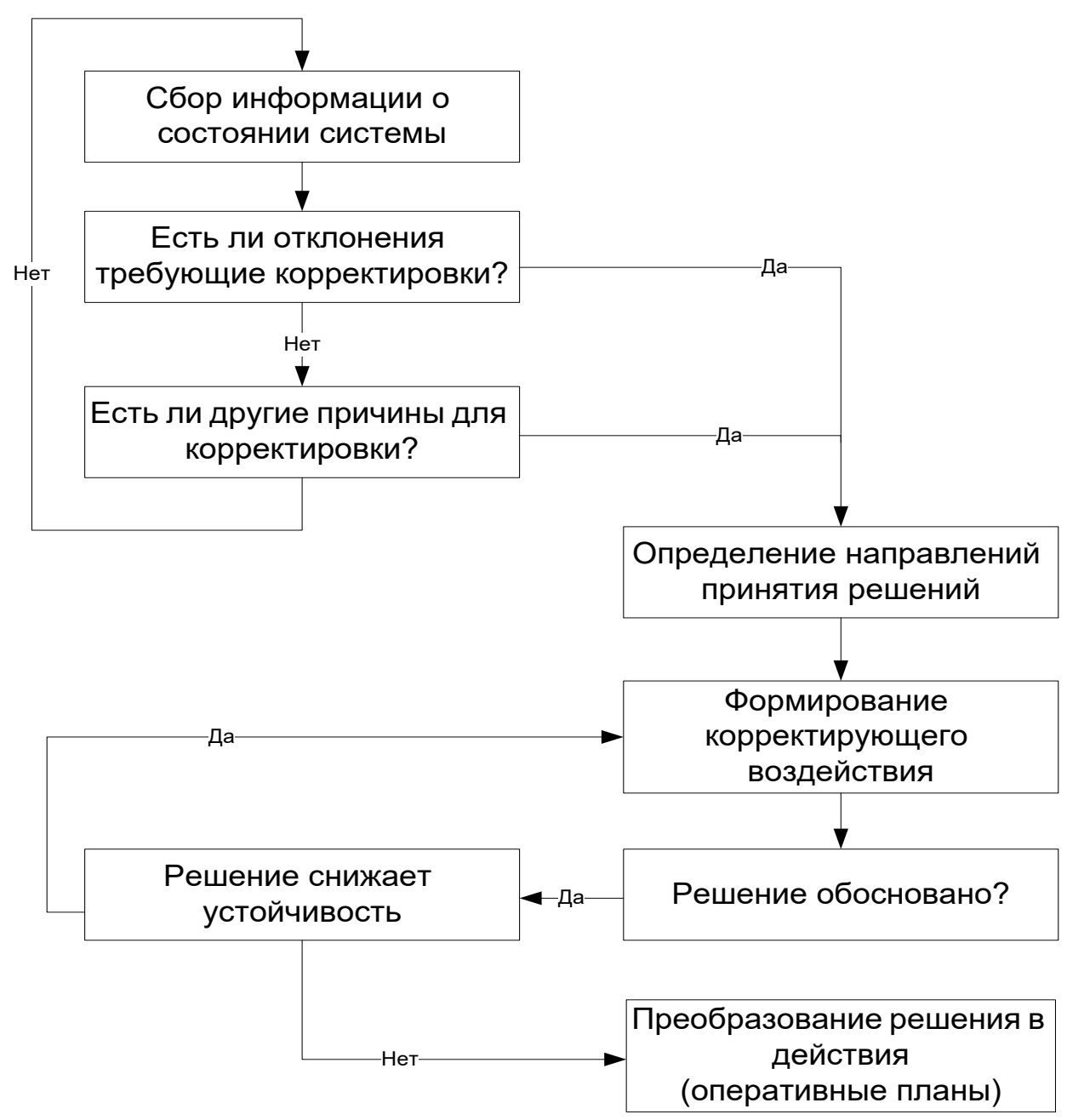

Рисунок 1. Алгоритм управления «по отклонениям»

вами, у управленца появляется необходимость оцифровать свои «желания» в конкретные значения параметров. Формирование самого набора параметров не является целью настоящей статьи, однако оно дает понимание, каким образом применение АОМ влияет на данный этап алгоритма управления. При этом параметры используются не только для отражения желаемого состояния системы, но и желаемых результатов деятельности каждой подсистемы. Значимым этапом реализации данного этапа будет являться формирование базиса, то есть того набора ресурсов, действий, агентов, институтов, которые впоследствии будут оцениваться на следующих этапах алгоритма управления.

На этапе сбора информации о состоянии системы AOM задает набор необходимых данных, формы их фиксации и методику оценки. Субъект управления получает информацию какие имен- но данные необходимо собрать:

- Параметры ресурсной базы,

- Параметры деятельности,

- Параметры информационной среды,

- Параметры агентов - участников корпоративных отношений,

- Параметры институциональной среды,

- Параметры внешней среды.

Выше представлены группы параметров, в каждой из которых есть ряд задаваемых (тех, которые непосредственно оцениваются субъектом управления). Другие являются расчетными - они вычисляются по алгоритмам модели на основе значений задаваемых параметров. Этап сбора информации о состоянии системы сводится к выявлению значений параметров, определенных ранее в модели при построении эталонного состояния системы. АОМ является имитационным, оно позволяет прогнозировать 
поведение системы на основе входных параметров. Это позволяет при идентификации текущего состояния уже на этом этапе проиграть поведение системы и выявить также прогнозируемое состояние в течение заданного количества периодов дискретизации.

На этапе выявления отклонений, требующих корректировки, субъект управления, использующий АОМ, исходя из информации, зафиксированной на предыдущем этапе, может выявить, во-первых, несоответствие текущего состояния эталонному, что выражается в значениях их параметров. Данные несоответствия будут являться направлениями принятия решений. Так задача управленца, использующего АOM, будет заключаться в формировании и принятии решений, которые устранят или минимизируют выявленные отклонения в значениях параметров. Помимо этого, ранее уже было отмечено, что на этапе сбора информации АОМ позволяет прогнозировать поведение системы и ее будущее состояние. Субъект управления получает возможность выявить вероятное поведение участников корпоративных отношений, проявление активности, информационные и ресурсные потоки. Вследствие этого, появляется информация не только о текущих, но и о будущих отклонениях от эталонного либо планируемого состояния и их причинах, что также является отклонениями, требующими корректировок. Стоит отметить, что АОМ в некоторых ситуациях позволяет выявить и те отклонения, которые не требуют корректировок. Так, нежелательное поведение агента в текущий момент может привести к положительной активности других агентов, что в целом, будет полезно для системы и обеспечит через некоторое время необходимый вектор поведения организации.

Другие причины, требующие внесения корректировок выявляются субъектом на основе факторов, не учтенных в модели. На этом этапе применение АОМ нецелесообразно, однако в результате выявления неучтенных факторов, модель может быть скорректирована для повышения ее адекватности рассматриваемому объекту моделирования.

При определении направлений принятия решений субъекту управления необходимо на основе полученных ранее данных об отклонениях, требующих корректировок, принять решения о том, значения каких именно параметров необходимо изменить и каким образом. Изме- нять значения всех несоответствующих параметров - задача маловероятно реализуемая, так как отклонений может быть много и значение ряда параметров не может быть изменено по желанию субъекта управления. Так, нельзя напрямую изменить вероятность реализации определенного действия агентом, однако можно создать институты, которые приведут к необходимой корректировке его поведения. AOM задает и формализует связи параметров. Понимание закономерностей ее построения позволяет управленцу выявить те параметры, изменение значений которых приведет к оптимальному устранению отклонений.

Формирование корректирующих воздействий в определенных ранее направлениях принятия решений производится управленцем, здесь модель неприменима. Субъект управления разрабатывает решения, как ему изменить значение заданного параметра: привлечь ресурс извне, произвести внутри системы, изменить параметры институтов либо информационных групп и так далее. Важно, что для проверки решений в АОМ они должны быть оцифрованы. Недостаточно принять решение в виде просто найма специалиста. Необходимо оценить сколько и каких ресурсов появится в системе, как изменятся параметры институциональной и информационной среды, как изменится вектор поведения системы и так далее.

При проверке сформированных решений, они вводятся в модель как изменения параметров текущего состояния системы. АОМ проигрывает поведение системы и позволяет выявить будущие риски при принятии того либо иного решения. Часть параметров модели являются статичными в рамках модели, другая часть - динамичные. Изменение входных параметров обуславливает поведение агентов на заданном количестве периодов дискретизации и соответствующее такому поведению изменения состояния системы, что выражается в корректировке значений динамичных параметров. Так, проигрывая поведение системы в результате принятия альтернативных решений субъект управления получает возможность оценить риски и преимущества, сложные для идентификации без соответствующих расчетов. Значительный объем данных не позволяет своевременно производить такие расчеты без применения соответствующих инструментов, что повышает значимость применения AOM в системах управ- 
ления. Использование данного инструмента на этом этапе позволяет определить и принять наиболее обоснованное решение с учетом его эффекта для текущей и будущей реализации целевой функции. В результате проигрывания поведения системы могут быть выявлены потенциальные риски, что позволяет сформировать комплементарные мероприятия по их минимизации, превентивно устраняя возможные негативные последствия различных управленческих решений.

На этапе преобразования решений в мероприятия AMO неприменима, однако субъект управления должен контролировать соответствие изменения значений параметров системы прогнозным, которые могут быть связаны с некорректной оценкой входных параметров либо неучетом значимых элементов (институтов, ключевых ресурсов, каналов передачи инфор- мации) при построении модели системы. При выявлении несоответствий модель необходимо корректировать.

АОМ находит применение на большинстве этапов алгоритма управления, повышая обоснованность решений, формализуя и направляя сам процесс принятия управленческих решений. Структурируем место АОМ в системе управления организацией в Таблице 1.

Применение АОМ в системе управления организации позволяет повысить качество управления, обоснованность решений, формализует и делает прозрачным процесс их принятия. В условиях развития этого инструмента, компьютерных технологий, цифровизации экономики и других сфер деятельности представляется крайне перспективным и полезным развитие АOM для задач управления организациями.

Таблица 1. Место АОМ в системе управления.

\begin{tabular}{|c|c|c|}
\hline № & Этап алгоритма & Место модели \\
\hline - & $\begin{array}{l}\text { Формирование эталонного } \\
\text { состояния }\end{array}$ & $\begin{array}{l}\text { Формализация эталонного состояния в виде значений конкретных } \\
\text { параметров, вводимых в модель. } \\
\text { Формирование базиса параметров организации }\end{array}$ \\
\hline 1 & $\begin{array}{l}\text { Сбор информации о состоянии } \\
\text { системы, }\end{array}$ & $\begin{array}{l}\text { Модель обеспечивает перечень необходимых для сбора данных, } \\
\text { формы их фиксации, методику перевода в цифровые значения. } \\
\text { Модель позволяет выявить будущее состояние системы на основы } \\
\text { текущих значений параметров. }\end{array}$ \\
\hline 2 & $\begin{array}{l}\text { Есть ли отклонения требующие } \\
\text { корректировки? }\end{array}$ & $\begin{array}{l}\text { Формализованная и оцифрованная информация об эталонном, } \\
\text { плановом и текущем состоянии системы четко отражает отклонения } \\
\text { в виде несоответствий значений параметров. } \\
\text { Также АОМ позволяет идентифицировать будущие отклонения для } \\
\text { принятия превентивных решений. }\end{array}$ \\
\hline 3 & $\begin{array}{l}\text { Есть ли другие причины для } \\
\text { корректировки? }\end{array}$ & - \\
\hline 4 & $\begin{array}{l}\text { Определения направлений } \\
\text { принятия решений, }\end{array}$ & $\begin{array}{l}\text { АОМ задает взаимосвязи параметров, позволяя субъекту управле- } \\
\text { ния определить те их них, которые оптимальным образом могут } \\
\text { привести к устранению либо минимизации выявленных отклоне- } \\
\text { ний. }\end{array}$ \\
\hline 5 & $\begin{array}{l}\text { Формирование корректирую- } \\
\text { щего воздействия, }\end{array}$ & $\begin{array}{l}\text { Модель обуславливает необходимость перевода решений в цифро- } \\
\text { вые изменения значений параметров, что обеспечивает необходи- } \\
\text { мость оценить различные факторы при его принятии. }\end{array}$ \\
\hline 6 & $\begin{array}{l}\text { Проверка решения на обосно- } \\
\text { ванность и влияние на устой- } \\
\text { чивость, }\end{array}$ & $\begin{array}{l}\text { Модель позволяет смоделировать поведение системы и ее изме- } \\
\text { нение при принятии каждого из решений, что дает возможность } \\
\text { принять наиболее обоснованное, исходя из текущего состояния } \\
\text { системы, решение. } \\
\text { Также моделирование поведения системы позволяет оценить } \\
\text { возможные риски при принятии решений и сформировать превен- } \\
\text { тивные комплементарные мероприятия, что повышает качество } \\
\text { принимаемых решений. }\end{array}$ \\
\hline 7 & $\begin{array}{l}\text { Преобразование решения } \\
\text { в действия. }\end{array}$ & - \\
\hline
\end{tabular}




\section{Библиографический список}

1. Агеева А.Ф. Социально-природные и социо-эколого-экономические модели, созданные с помощью метода агентного моделирования / Агеева А.Ф. // Вестник Российского экономического университета имени Г. В. Плеханова. - 2018. - № 4 (100).- С. 100-115.

2. Акопов А. С. Разработка адаптивного генетического оптимизационного алгоритма с использованием методов агентного моделирования / Акопов А.С., Бекларян А.Л., Хачатрян Н.К., Фомин А. В. // Информационные технологии. - 2018. Т. 24. - № 5.- С. 321-329.

3. Багрин П.П. Возможность имитационного моделирования корпоративных систем / Багрин П.П. // Теоретическая и прикладная экономика.- 2016. - № 1.- C. 1-11. DOI: 10.7256/2409-8647.2016.1.17763 URL: http://e-notabene.ru/etc/article_17763.html,, 0,48 п.л ыч

4. Бекларян Г.Л. Агентное моделирование межрегиональных железнодорожных грузоперевозок / Бекларян Г. Л., Акопов А. С. // Аудит и финансовый анализ.-2019.- № 1.- С. 39-54.

5. Бекларян Г.Л. Агентное моделирование региональных экономических систем / Бекларян Г.Л., Акопов А.С. // Вестник ЦЭМИ.-2019.- № 1.- С. 14.

6. Гайкова Л.В. Агентное моделирование и поведенческая экономика как прикладной инструмент стратегии потребительского рынка / Гайкова Л. В. // В сборнике: Могущество Сибири будет прирастать!?. Материалы международного научного форума «Образование и предпринимательство в Сибири: направления взаимодействия и развитие регионов».-2017.-С. 36.

7. Самосудов М..В. Развитие теории корпоративного управления. /Самосудов М.В. // Диссертация на соискание научной степени доктора экономических наук. - 2011. - 440 с.

8. Сорокин А. А. Агентная модель выбора пассажирами вида транспорта в среде имитационного моделирования Anylogic / Сорокин А.А.// Свидетельство о регистрации программы для ЭВM RU 2015617032, 26.06.2015. Заявка № 2015612135 от 25.03.2015.

9. Филяк П.Ю. Обеспечение информационной безопасности с помощью систем имитационного моделирования «ORA» / Филяк П.Ю., Ермолин А.Н., Уразов О.М. // В сборнике: Фундаментально-прикладные проблемы безопасности, живучести, надежности, устойчивости и эффективности систем. Материалы III международной научно-практической конференции, посвящённой 110-летию со дня рождения академика Н.А. Пилюгина. - 2019.С. - 154-159. 